\title{
The Lattice of Intuitionistic Fuzzy Filters in Residuated Lattices
}

\author{
Zhen Ming Ma \\ School of Science, Linyi University, Linyi, Shandong 276005, China \\ Correspondence should be addressed to Zhen Ming Ma; dmgywto@126.com \\ Received 6 November 2013; Accepted 10 April 2014; Published 29 April 2014 \\ Academic Editor: Ch. Tsitouras \\ Copyright (C) 2014 Zhen Ming Ma. This is an open access article distributed under the Creative Commons Attribution License, \\ which permits unrestricted use, distribution, and reproduction in any medium, provided the original work is properly cited.
}

The notion of tip-extended pair of intuitionistic fuzzy filters is introduced by which it is proved that the set of all intuitionistic fuzzy filters in a residuated lattice forms a bounded distributive lattice.

\section{Introduction}

Nowadays, it is generally accepted that in fuzzy logic the algebraic structure should be a residuated lattice which was introduced by Ward and Dilworth [1]. Some other logical algebras such as MTL-algebras [2], BL-algebras [3], MValgebras [4], G-algebras, П-algebras, and NM-algebras [2], which are also called $R_{0}$-algebras [5], are all able to be considered particular classes of residuated lattices. (For details, see, e.g., [6].)

Filters are an important tool to study these logical algebras and the completeness of the corresponding nonclassical logics. On the one hand, filters are closely related to congruence relations with which one can associate quotient algebras [7]; on the other hand, various filters correspond to various sets of provable formula $[3,4]$. A filter is also called a deductive system in BL-algebras [8]. It has been widely investigated in residuated lattices [7, 9-11] and particular residuated lattices $[2,3,6,8,12-15]$.

Since Rosenfeld [16] applied the notion of fuzzy sets [17] to abstract algebra and introduced the notion of fuzzy subgroups, the literature of various fuzzy algebraic concepts has been growing very rapidly [18]. In particular, in [19-21], the notion of tip-extended pair of fuzzy sets was introduced to investigate the lattices of all fuzzy normal subgroups and $L$-ideals.

The notion of fuzzy filters was introduced, and some properties of them were obtained [22-24]. Moreover, based on the notion of intuitionistic fuzzy sets (IFS) proposed by Atanassov [25], the concept of the intuitionistic fuzzy filter in BL-algebras was introduced in [26]. However, the study of residuated lattices from the point of lattice theory is less frequent.

In this paper, the intuitionistic fuzzy filter theory in residuated lattices is developed. This paper is organized as follows: in Section 2, some basic concepts and properties of intuitionistic fuzzy sets and intuitionistic fuzzy filters in residuated lattices are recalled. In Section 3, by introducing the notion of tip-extended pair of intuitionistic fuzzy filters, it is proved that the set of all intuitionistic fuzzy filters forms a bounded distributive lattice. The last section concludes this paper.

\section{Preliminaries}

The concepts of residuated lattices and intuitionistic fuzzy filters will be extensively used in the sequel. Therefore, we recall their definitions and summarize their main properties.

Let $U \neq \emptyset$. A mapping $f: U \rightarrow[0,1]$ is called a fuzzy set [17]. Let $f$ and $g$ be fuzzy sets on $U$. Then tip-extended pair of $f$ and $g[19,20]$ can be defined by

$$
\begin{aligned}
& f^{g}(x)= \begin{cases}f(x), & x \neq 1, \\
f(1) \vee g(1), & x=1,\end{cases} \\
& g^{f}(x)= \begin{cases}g(x), & x \neq 1, \\
g(1) \vee f(1), & x=1 .\end{cases}
\end{aligned}
$$

Let $u_{A}, v_{A}: U \rightarrow[0,1]$ be two fuzzy sets satisfying $0 \leq$ $u_{A}(x)+v_{A}(x) \leq 1$ for all $x \in U$. Then $A=\left(u_{A}, v_{A}\right)$ is called an intuitionistic fuzzy set [25] (or equivalently denoted by 
$\left.A=\left\{\left\langle x, u_{A}(x), v_{A}(x)\right\rangle \mid x \in U\right\}\right)$. The family of all intuitionistic fuzzy sets on $U$ will be denoted by $\operatorname{IFS}(U)$.

Basic operations on intuitionistic fuzzy sets are defined in the following way.

Let $A, B \in \operatorname{IFS}(U)$. One has

$$
\begin{gathered}
A \cap B=\left(u_{A} \wedge u_{B}, v_{A} \vee v_{B}\right), \\
A \cup B=\left(u_{A} \vee u_{B}, v_{A} \wedge v_{B}\right), \\
A \subseteq B \quad \text { iff } u_{A} \leq u_{B}, v_{A} \geq v_{B}, \\
A \supseteq B \quad \text { iff } A \subseteq B, \\
A=B \quad \text { iff } A \supseteq B, A \subseteq B .
\end{gathered}
$$

Definition 1 (see [3]). A residuated lattice is an algebra $L=(L, \wedge, \vee, \otimes, \rightarrow, 0,1)$ such that $(L, \wedge, \vee, 0,1)$ is a bounded lattice with the least element 0 and the greatest element 1 , $(L, \otimes, 1)$ is a commutative monoid, and $(\otimes, \rightarrow)$ forms an adjoint pair; that is, $x \otimes y \leq z$ if and only if $x \leq y \rightarrow z$ for all $x, y, z \in L$.

A nonempty subset $F$ of $L$ is called a filter of $L$ if (i) $\forall x, y \in$ $F, x \otimes y \in F$; (ii) $\forall x \in F, y \in L, x \leq y$ implies $y \in F$ or, equivalently, (iii) $1 \in F$; and (iv) $\forall x \in F, y \in L, x \rightarrow y \in F$ implies $y \in F$.

The following alternative definitions of intuitionistic fuzzy filters were proved in [26], but they can be similarly verified in residuated lattices.

Definition 2. Let $A \in \operatorname{IFS}(L)$. Then $A$ is called an intuitionistic fuzzy filter if

(1) $u_{A}(x) \leq u_{A}(1), v_{A}(x) \geq v_{A}(1)$ for all $x \in L$;

(2) $u_{A}(x) \wedge u_{A}(x \rightarrow y) \leq u_{A}(y)$ for all $x, y \in L$;

(3) $v_{A}(x) \vee v_{A}(x \rightarrow y) \geq v_{A}(y)$ for all $x, y \in L$.

The set of all intuitionistic fuzzy filters on a residuated lattice $L$ will be denoted by $\operatorname{IFF}(L)$.

Theorem 3. Let $A \in \operatorname{IFS}(L)$. Then $A$ is an intuitionistic fuzzy filter if and only if $x \otimes y \leq z$ implies $u_{A}(x) \wedge u_{A}(y) \leq u_{A}(z)$ and $v_{A}(x) \vee v_{A}(y) \geq v_{A}(z)$ for all $x, y, z \in L$.

Theorem 4. Let $A \in \operatorname{IFS}(L)$. Then $A$ is an intuitionistic fuzzy filter if and only if the following assertions hold:

(1) $x \leq y$ implies $u_{A}(x) \leq u_{A}(y)$ and $v_{A}(x) \geq v_{A}(y)$ for all $x, y \in L$;

(2) $u_{A}(x) \wedge u_{A}(y) \leq u_{A}(x \otimes y)$ and $v_{A}(x) \vee v_{A}(y) \geq v_{A}(x \otimes$ y) for all $x, y \in L$.

\section{Lattice of Intuitionistic Fuzzy Filters}

In this section, we mainly investigate the lattice of all intuitionistic fuzzy filters by introducing the notion of tipextended pair of intuitionistic fuzzy sets.

The following lemma is obvious but necessary.

Lemma 5. Let $A, B$ be intuitionistic fuzzy filters of $L$. Then so is $A \cap B$.
For $A \in \operatorname{IFS}(L)$, the intersection of all intuitionistic fuzzy filters containing $A$ is called the generated intuitionistic fuzzy filter by $A$, denoted as $\langle A\rangle$.

Theorem 6. Let $A \in \operatorname{IFS}(L)$. Define a new intuitionistic fuzzy set $B$ by $B=\left(u_{B}, v_{B}\right)$ where

$$
\begin{gathered}
u_{B}(x)=\bigvee_{\substack{a_{1} \otimes \cdots \otimes a_{n} \leq x \\
a_{i} \in L, n \in N}}\left\{u_{A}\left(a_{1}\right) \wedge \cdots \wedge u_{A}\left(a_{n}\right)\right\}, \\
v_{B}(x)=\bigwedge_{\substack{a_{1} \otimes \cdots \otimes a_{n} \leq x \\
a_{i} \in L, n \in N}}\left\{v_{A}\left(a_{1}\right) \vee \cdots \vee u_{A}\left(a_{n}\right)\right\}
\end{gathered}
$$

for all $x \in L$. Then $B=\langle A\rangle$.

Proof. We complete the proof by two steps. Firstly, we verify that $B$ is an intuitionistic fuzzy filter. For all $x, y \in L$, such that $x \leq y$, the definition of $B$ yields that $u_{B}(x) \leq u_{B}(y)$ and $v_{B}(x) \geq v_{B}(y)$. For all $x, y \in L$, we have

$$
\begin{aligned}
& u_{B}(x) \wedge u_{B}(y) \\
& =\bigvee_{\substack{a_{1} \otimes \cdots \otimes a_{n} \leq x \\
a_{i} \in L, n \in N}}\left\{u_{A}\left(a_{1}\right) \wedge \cdots \wedge u_{A}\left(a_{n}\right)\right\} \\
& \wedge \bigvee_{\substack{b_{1} \otimes \cdots \otimes b_{m} \leq y \\
b_{i} \in L, m \in N}}\left\{u_{A}\left(b_{1}\right) \wedge \cdots \wedge u_{A}\left(b_{m}\right)\right\} \\
& =\bigvee_{\substack{a_{1} \otimes \cdots \otimes a_{n} \leq x \\
a_{i} \in L, n \in N}} \bigvee_{\substack{b_{1} \otimes \cdots \otimes b_{m} \leq y \\
b_{i} \in L, m \in N}}\left\{u_{A}\left(a_{1}\right) \wedge \cdots \wedge u_{A}\left(a_{n}\right)\right. \\
& \left.\wedge u_{A}\left(b_{1}\right) \wedge \cdots \wedge u_{A}\left(b_{m}\right)\right\} \\
& \leq \bigvee_{\substack{c_{1} \otimes \cdots \otimes c_{n} \leq x \otimes y \\
c_{i} \in L, k \in N}}\left\{u_{A}\left(c_{1}\right) \wedge \cdots \wedge u_{A}\left(c_{k}\right)\right\} \\
& =u_{B}(x \otimes y), \\
& v_{B}(x) \vee v_{B}(y) \\
& =\bigwedge_{\substack{a_{1} \otimes \cdots \otimes a_{n} \leq x \\
a_{i} \in L, n \in N}}\left\{u_{A}\left(a_{1}\right) \vee \cdots \vee u_{A}\left(a_{n}\right)\right\} \\
& \vee \bigwedge_{\substack{b_{1} \otimes \cdots \otimes b_{m} \leq y \\
b_{i} \in L, m \in N}}\left\{u_{A}\left(b_{1}\right) \vee \cdots \vee u_{A}\left(b_{m}\right)\right\} \\
& =\bigwedge_{\substack{a_{1} \otimes \cdots \otimes a_{n} \leq x \\
a_{i} \in L, n \in N}} \bigwedge_{\substack{b_{1} \otimes \cdots \otimes b_{m} \leq y \\
b_{i} \in L, m \in N}}\left\{u_{A}\left(a_{1}\right) \vee \cdots \vee u_{A}\left(a_{n}\right)\right. \\
& \left.\vee u_{A}\left(b_{1}\right) \vee \cdots \vee u_{A}\left(b_{m}\right)\right\} \\
& \geq \bigwedge_{\substack{c_{1} \otimes \cdots \otimes c_{n} \leq x \otimes y \\
c_{i} \in L, k \in N}}\left\{u_{A}\left(c_{1}\right) \vee \cdots \vee u_{A}\left(c_{k}\right)\right\} \\
& =u_{B}(x \otimes y) \text {. }
\end{aligned}
$$

Thus $B$ is an intuitionistic fuzzy filter. 
Secondly, let $C$ be an intuitionistic fuzzy filter such that $C \supseteq A$. By the definition of intuitionistic fuzzy filter, it holds that

$$
\begin{aligned}
u_{B}(x) & =\bigvee_{\substack{a_{1} \otimes \cdots \otimes a_{n} \leq x \\
a_{i} \in L, n \in N}}\left\{u_{A}\left(a_{1}\right) \wedge \cdots \wedge u_{A}\left(a_{n}\right)\right\} \\
& \leq \bigvee_{\substack{a_{1} \otimes \cdots \otimes a_{n} \leq x \\
a_{i} \in L, n \in N}}\left\{u_{C}\left(a_{1}\right) \wedge \cdots \wedge u_{C}\left(a_{n}\right)\right\} \\
& \leq \bigvee_{\substack{a_{1} \otimes \cdots \otimes a_{n} \leq x \\
a_{i} \in L, n \in N}}\left\{u_{C}\left(a_{1} \otimes \cdots \otimes a_{n}\right)\right\} \\
& \leq u_{C}(x), \\
v_{B}(x) & =\bigwedge_{\substack{a_{1} \otimes \cdots \otimes a_{n} \leq x \\
a_{i} \in L, n \in N}}\left\{u_{A}\left(a_{1}\right) \vee \cdots \vee u_{A}\left(a_{n}\right)\right\} \\
& \geq \bigwedge_{\substack{a_{1} \otimes \cdots \otimes a_{n} \leq x \\
a_{i} \in L, n \in N}}\left\{u_{C}\left(a_{1}\right) \vee \cdots \vee u_{C}\left(a_{n}\right)\right\} \\
& \geq \bigwedge_{\substack{a_{1} \otimes \cdots \otimes a_{n} \leq x \\
a_{i} \in L, n \in N}}\left\{u_{C}\left(a_{1} \otimes \cdots \otimes a_{n}\right)\right\} \\
& \geq u_{C}(x),
\end{aligned}
$$

\begin{tabular}{|c|c|c|c|}
\hline$\otimes$ & $\begin{array}{llll}0 & a & b & 1\end{array}$ & $\rightarrow$ & $\begin{array}{lll}0 & a & b\end{array}$ \\
\hline 0 & $\begin{array}{llll}0 & 0 & 0 & 0\end{array}$ & 0 & $\begin{array}{lll}1 & 1 & 1\end{array}$ \\
\hline$a$ & $\begin{array}{lllllll}0 & 0 & a & a\end{array}$ & $a$ & $\begin{array}{lll}a & 1 & 1\end{array}$ \\
\hline$b$ & $0 \quad a b b$ & $b$ & $\begin{array}{lll}0 & a & 1\end{array}$ \\
\hline 1 & $\begin{array}{llll}0 & a & b & 1\end{array}$ & 1 & $\begin{array}{lll}0 & a & b\end{array}$ \\
\hline
\end{tabular}

and hence $B \subseteq C$. Thus $B=\langle A\rangle$.

Example 7. Let $L=\{0, a, b, 1\}$ with $0<a<b<1$. The operations $\otimes$ and $\rightarrow$ are defined as

Define $u_{A}, v_{A}: L \rightarrow[0,1]$ as $u_{A}(0)=0.2, v_{A}(0)=$ $0.7, u_{A}(a)=0.5, v_{A}(a)=0.45, u_{A}(b)=0.6, v_{A}(b)=$ $0.4, u_{A}(1)=0.8$, and $v_{A}(1)=0.15$. Since $0.5=u_{A}(a) \wedge$ $u_{A}(a) \nless u_{A}\left(a^{2}\right)=0.2, f$ is not a fuzzy filter. It is routine to verify that $\langle A\rangle$ is an intuitionistic fuzzy filter, where $u_{\langle A\rangle}(0)=u_{\langle A\rangle}(a)=0.5, v_{\langle A\rangle}(0)=v_{\langle A\rangle}(a)=0.4, u_{\langle A\rangle}(b)=$ $0.6, v_{\langle A\rangle}(b)=0.3, u_{\langle A\rangle}(1)=0.8$, and $v_{\langle A\rangle}(1)=0.15$ from the above theorem.

Lemma 8. Let $a, b, s, t \in[0,1]$ such that $0 \leq a+b \leq 1$ and $0 \leq s+t \leq 1$. Then $0 \leq a \vee s+b \wedge t \leq 1$.

Proof. Not losing the generality, we assume that $a \leq s$. Then $a \vee s+b \wedge t \leq s+t \leq 1$. It is obvious that $0 \leq a \vee s+b \wedge t$. Thus it holds that $0 \leq a \vee s+b \wedge t \leq 1$.
Theorem 9. Let $A$ be an intuitionistic fuzzy filter of $L$ and for all $t, s \in[0,1]$ such that $0 \leq t+s \leq 1$. Then $A^{t, s}=\left(u_{A}^{t}, v_{A}^{s}\right)$ is an intuitionistic fuzzy filter, where

$$
\begin{aligned}
& u_{A}^{t}(x)= \begin{cases}u_{A}(x), & x \neq 1, \\
u_{A}(1) \vee t, & x=1,\end{cases} \\
& v_{A}^{s}(x)= \begin{cases}v_{A}(x), & x \neq 1, \\
v_{A}(1) \wedge s, & x=1 .\end{cases}
\end{aligned}
$$

Proof. It follows from Lemma 8 that $A^{t, s} \in \operatorname{IFS}(L)$. Now we prove that $A^{t, s}$ is an intuitionistic fuzzy filter.

If $x \leq y$, we consider the following two cases:

Case $1(y=1)$. It is obvious that $u_{A}^{t}(x) \leq u_{A}^{t}(1)=u_{A}^{t}(y)$, $v_{A}^{t}(x) \geq v_{A}^{t}(1)=v_{A}^{t}(y)$.

Case $2(y \neq 1)$. The definition of $A^{t, s}$ leads that $u_{A}^{t}(x)=$ $u_{A}(x) \leq u_{A}(y)=u_{A}^{t}(y), v_{A}^{t}(x)=v_{A}(x) \geq v_{A}(y)=v_{A}^{t}(y)$.

Thus $u_{A}^{t}(x) \leq u_{A}^{t}(y), v_{A}^{t}(x) \geq v_{A}^{t}(y)$.

Let $x, y \in L$. We consider the following two cases:

Case $1(x \otimes y=1)$. If $x=y=1$, it is obvious that $u_{A}^{t}(x) \wedge$ $u_{A}^{t}(y) \leq u_{A}^{t}(x \otimes y), v_{A}^{t}(x) \vee y_{A}^{t}(x) \geq v_{A}^{t}(x \otimes y)$.

If $x=1, y \neq 1$ or $x \neq 1, y=1$, it is a contradiction.

If $x \neq 1, y \neq 1$, it holds that $u_{A}^{t}(x) \wedge u_{A}^{t}(y)=u_{A}(x) \wedge$ $u_{A}(y) \leq u_{A}(x \otimes y)=u_{A}^{t}(x \otimes y), v_{A}^{t}(x) \vee y_{A}^{t}(x)=v_{A}(x) \vee$ $y_{A}(x) \geq v_{A}(x \otimes y)=v_{A}^{t}(x \otimes y)$.

Case $2(x \otimes y \neq 1)$. If $x=y=1$, it is a contradiction.

If $x=1, y \neq 1$ or $x \neq 1, y=1$, it is obvious that $u_{A}^{t}(x) \wedge$ $u_{A}^{t}(y) \leq u_{A}^{t}(x \otimes y), v_{A}^{t}(x) \vee y_{A}^{t}(x) \geq v_{A}^{t}(x \otimes y)$.

If $x \neq 1, y \neq 1$, we have $u_{A}^{t}(x) \wedge u_{A}^{t}(y)=u_{A}(x) \wedge u_{A}(y) \leq$ $u_{A}(x \otimes y)=u_{A}^{t}(x \otimes y), v_{A}^{t}(x) \vee y_{A}^{t}(x)=v_{A}(x) \vee y_{A}(x) \geq$ $v_{A}(x \otimes y)=v_{A}^{t}(x \otimes y)$.

All in all, it yields that $u_{A}^{t}(x) \wedge u_{A}^{t}(y)=u_{A}(x) \wedge u_{A}(y) \leq$ $u_{A}(x \otimes y)=u_{A}^{t}(x \otimes y), v_{A}^{t}(x) \vee y_{A}^{t}(x)=v_{A}(x) \vee y_{A}(x) \geq$ $v_{A}(x \otimes y)=v_{A}^{t}(x \otimes y)$.

Thus $A^{t, s}$ is an intuitionistic fuzzy filter.

For given $A, B \in \operatorname{IFS}(L)$, the operation $\widetilde{\star}$ is defined by

$$
A \widetilde{\star} B=\left(u_{A} \widetilde{\otimes} u_{B}, v_{A} \widetilde{\odot} v_{B}\right),
$$

where

$$
\begin{aligned}
& u_{A} \widetilde{\otimes} u_{B}(x)=\bigvee_{y \otimes z \leq x}\left\{u_{A}(y) \wedge u_{B}(z)\right\}, \\
& v_{A} \widetilde{\odot} v_{B}(x)=\bigwedge_{y \otimes z \leq x}\left\{v_{A}(y) \vee v_{B}(z)\right\} .
\end{aligned}
$$

Furthermore, the tip-extended pair for intuitionistic fuzzy sets $A$ and $B$ are defined by

$$
A^{B}=\left(u_{A}^{u_{B}}, v_{A}^{v_{B}}\right)
$$


where

$$
\begin{aligned}
& u_{A}^{u_{B}}(x)= \begin{cases}u_{A}(x), & x \neq 1, \\
u_{A}(1) \vee u_{B}(1), & x=1,\end{cases} \\
& v_{A}^{v_{B}}(x)= \begin{cases}v_{A}(x), & x \neq 1, \\
v_{A}(1) \wedge v_{B}(1), & x=1,\end{cases} \\
& B^{A}=\left(u_{B}^{u_{A}}, v_{B}^{v_{A}}\right),
\end{aligned}
$$

where

$$
\begin{aligned}
u_{B}^{u_{A}}(x) & = \begin{cases}u_{B}(x), & x \neq 1, \\
u_{B}(1) \vee u_{A}(1), & x=1,\end{cases} \\
v_{B}^{v_{A}}(x) & = \begin{cases}v_{B}(x), & x \neq 1, \\
v_{B}(1) \wedge v_{A}(1), & x=1 .\end{cases}
\end{aligned}
$$

Theorem 10. Let $A, B \in \operatorname{IFF}(L)$. Then $A^{B} \widetilde{\star} B^{A} \in \operatorname{IFF}(L)$.

Proof. It is obvious that $u_{A}^{u_{B}} \widetilde{\otimes} u_{B}^{u_{A}}$ is order-preserving, and $v_{A}^{v_{B}} \widetilde{\rho} v_{B}^{v_{A}}$ is order-reserving. For all $x, y \in L$, we have

$$
\begin{aligned}
u_{A}^{u_{B}} \widetilde{\otimes} & u_{B}^{u_{A}}(x \otimes y) \\
& =\bigvee_{u \otimes v \leq x \otimes y}\left\{u_{A}^{u_{B}}(u) \wedge u_{B}^{u_{A}}(v)\right\} \\
& \geq \bigvee_{p \otimes q \leq x}\left\{u_{A}^{u_{B}}(p \otimes r) \wedge u_{B}^{u_{A}}(q \otimes s)\right\} \\
& \geq \bigvee_{r \otimes s \leq y \leq x}\left\{u_{A}^{u_{B}}(p) \wedge u_{A}^{u_{B}}(r) \wedge u_{B}^{u_{A}}(q) \wedge u_{B}^{u_{A}}(s)\right\} \\
& =\bigvee_{p \otimes q \leq y \leq x}\left\{u_{A}^{u_{B}}(p) \wedge u_{B}^{u_{A}}(q)\right\} \wedge \bigvee_{r \otimes s \leq y}\left\{u_{A}^{u_{B}}(r) \wedge u_{B}^{u_{A}}(s)\right\} \\
& =u_{A}^{u_{B}} \widetilde{\otimes} u_{B}^{u_{A}}(x) \wedge u_{A}^{u_{B}} \widetilde{\otimes u_{B}^{u_{A}}}(y), \\
v_{A}^{v_{B}} \widetilde{\odot} v_{B}^{v_{A}}(x \otimes y) & =\bigwedge_{u \otimes v \leq x \otimes y}\left\{v_{A}^{v_{B}}(u) \vee v_{B}^{v_{A}}(v)\right\} \\
& \leq \bigwedge_{p \otimes q \leq x}\left\{v_{A}^{v_{B}}(p \otimes r) \vee v_{B}^{v_{A}}(q \otimes s)\right\} \\
& \leq \bigwedge_{p \otimes q \leq y \leq x}\left\{v_{A}^{v_{B}}(p) \vee v_{A}^{v_{B}}(r) \vee v_{B}^{v_{A}}(q) \vee v_{B}^{v_{A}}(s)\right\} \\
& =\bigwedge_{p \otimes q \leq y \leq x}\left\{v_{A}^{v_{B}}(p) \vee v_{B}^{v_{A}}(q)\right\} \vee \bigwedge_{r \otimes s \leq y}\left\{v_{A}^{v_{B}}(r) \vee v_{B}^{v_{A}}(s)\right\} \\
& =v_{A}^{v_{B}} \widetilde{\odot} v_{B}^{v_{A}}(x) \vee v_{A}^{v_{B}} \widetilde{\odot} v_{B}^{v_{A}}(y),
\end{aligned}
$$

and hence $u_{A}^{u_{B}} \widetilde{\otimes} u_{B}^{u_{A}}(x \otimes y) \quad \geq \quad u_{A}^{u_{B}} \widetilde{\otimes} u_{B}^{u_{A}}(x) \wedge$ $u_{A}^{u_{B}} \widetilde{\otimes} u_{B}^{u_{A}}(y), v_{A}^{v_{B}} \widetilde{\odot} v_{B}^{v_{A}}(x \otimes y) \leq v_{A}^{v_{B}} \widetilde{\odot} v_{B}^{v_{A}}(x) \vee v_{A}^{v_{B}} \widetilde{\odot} v_{B}^{v_{A}}(y)$.

Thus $A^{B} \widetilde{\star} B^{A} \in \operatorname{IFF}(L)$.
Theorem 11. Let $A, B \in \operatorname{IFF}(L)$. Then $A^{B} \widetilde{\star} B^{A}=\langle A \cup B\rangle$.

Proof. It is easy to prove that $A, B \subseteq A^{B} \widetilde{\star} B^{A}$, and hence $A \cup$ $B \subseteq A^{B} \widetilde{\star} B^{A}$. Thus $\langle A \cup B\rangle \subseteq A^{B} \widetilde{\star} B^{A}$.

Assume that $C \in \operatorname{IFF}(L)$ such that $A \cup B \subseteq C$. If $x=1$, we have $u_{A}^{u_{B}} \widetilde{\otimes} u_{B}^{u_{A}}(1)=u_{A}(1) \vee u_{B}(1) \leq u_{C}(1), v_{A}^{v_{B}} \widetilde{\odot} v_{B}^{v_{A}}(1)=$ $v_{A}(1) \wedge v_{B}(1) \geq v_{C}(1)$. If $x<1$, it holds that

$$
\begin{aligned}
u_{A}^{u_{B}} \widetilde{\otimes} u_{B}^{u_{A}}(x) & \\
= & \bigvee_{y \otimes z \leq x}\left\{u_{A}^{u_{B}}(y) \wedge u_{B}^{u_{A}}(z)\right\} \\
& =\bigvee_{\substack{y \otimes z \leq x \\
y \neq 1, z \neq 1}}\left\{u_{A}^{u_{B}}(y) \wedge u_{B}^{u_{A}}(z)\right\} \vee \bigvee_{y \leq x}\left\{u_{A}(y)\right\} \vee \bigvee_{z \leq x}\left\{u_{B}(z)\right\} \\
& =\bigvee_{\substack{y \otimes z \leq x \\
y \neq 1, z \neq 1}}\left\{u_{A}(y) \wedge u_{B}(z)\right\} \vee \bigvee_{y \leq x}\left\{u_{A}(y)\right\} \vee \bigvee_{z \leq x}\left\{u_{B}(z)\right\} \\
& \leq \bigvee_{\substack{y \otimes z \leq x \\
y \neq 1, z \neq 1}}\left\{u_{C}(y) \wedge u_{C}(z)\right\} \vee \bigvee_{y \leq x}\left\{u_{C}(y)\right\} \vee \bigvee_{z \leq x}\left\{u_{C}(z)\right\} \\
& =\bigvee_{y \otimes z \leq x}\left\{u_{A}(y) \wedge u_{B}(z)\right\} \\
& \leq u_{C}(x), \\
v_{A}^{v_{B}} \widetilde{\odot} v_{B}^{v_{A}}(x) & =\bigwedge_{y \otimes z \leq x}\left\{v_{A}^{v_{B}}(y) \vee v_{B}^{v_{A}}(z)\right\} \\
& =\bigwedge_{\substack{y \otimes z \leq x \\
y \neq 1, z \neq 1}}\left\{v_{A}^{v_{B}}(y) \vee v_{B}^{v_{A}}(z)\right\} \wedge \bigwedge_{y \leq x}\left\{v_{A}(y)\right\} \wedge \bigwedge_{z \leq x}\left\{v_{B}(z)\right\} \\
& =\bigwedge_{\substack{y \otimes z \leq x \\
y \neq 1, z \neq 1}}\left\{v_{A}(y) \vee v_{B}(z)\right\} \wedge \bigwedge_{y \leq x}\left\{v_{A}(y)\right\} \wedge \bigwedge_{z \leq x}\left\{v_{B}(z)\right\} \\
& \geq \bigwedge_{\substack{y \otimes z \leq x \\
y \neq 1, z \neq 1}}\left\{v_{C}(y) \vee v_{C}(z)\right\} \wedge \bigwedge_{y \leq x}\left\{v_{C}(y)\right\} \wedge \bigwedge_{z \leq x}\left\{v_{C}(z)\right\} \\
& =v_{C}(x) \\
& \left.\bigwedge_{y}(y) v_{B}(z)\right\} \\
&
\end{aligned}
$$

It follows from Theorem 10 that $A^{B} \widetilde{\star} B^{A}=\langle A \cup B\rangle$.

Associating with the above results, we prove the main theorem here. For $A, B \in \operatorname{IFF}(L)$, the operations $\square$ and $\sqcup$ on $\operatorname{IFF}(L)$ are defined by

$$
A \sqcap B=A \cap B, \quad A \sqcup B=A^{B} \widetilde{\star} B^{A} .
$$

Theorem 12. $(\operatorname{IFF}(L), \sqcap, \sqcup, \emptyset, L)$ is a bounded distributive lattice.

Proof. We only verify the distributivity. Obviously, it holds that $C \sqcap(A \sqcup B) \supseteq(C \sqcap A) \sqcup(C \sqcap B)$, so we only prove 
that $C \sqcap(A \sqcup B) \subseteq(C \sqcap A) \sqcup(C \sqcap B)$. Assume that $x \in L$ for $u_{C} \wedge u_{A}^{u_{B}} \widetilde{\otimes} u_{B}^{u_{A}}(x) \leq\left(u_{C} \wedge u_{A}\right)^{u_{C} \wedge u_{B}} \widetilde{\otimes}\left(u_{C} \wedge u_{B}\right)^{u_{C} \wedge u_{A}}(x)$, we consider the following two cases.

Case $1(x=1)$. We have

$$
\begin{aligned}
u_{C} \wedge u_{A \sqcup B}(1) & =u_{C}(1) \wedge u_{A}^{u_{B}} \widetilde{\otimes} u_{B}^{u_{A}}(1) \\
& =u_{C}(1) \wedge\left(u_{A}(1) \vee u_{B}(1)\right) \\
& =\left(u_{C}(1) \wedge u_{A}(1)\right) \vee\left(u_{C}(1) \wedge u_{B}(1)\right) \\
& =\left(u_{C} \wedge u_{A}\right)^{u_{C} \wedge u_{B}} \widetilde{\otimes}\left(u_{C} \wedge u_{B}\right)^{u_{C} \wedge u_{A}}(1), \\
v_{C} \vee v_{A \sqcup B}(1) & =v_{C}(1) \vee v_{A}^{v_{B}} \widetilde{\odot} v_{B}^{v_{A}}(1) \\
& =v_{C}(1) \vee\left(v_{A}(1) \wedge v_{B}(1)\right) \\
& =\left(v_{C}(1) \vee v_{A}(1)\right) \wedge\left(v_{C}(1) \vee v_{B}(1)\right) \\
& =\left(v_{C} \vee v_{A}\right)^{v_{C} \vee v_{B}} \widetilde{\odot}\left(v_{C} \vee v_{B}\right)^{v_{C} \vee v_{A}}(1)
\end{aligned}
$$

Case $2(x \neq 1)$. It holds that

$$
\begin{aligned}
& u_{C}(x) \wedge u_{A}^{u_{B}} \widetilde{\otimes} u_{B}^{u_{A}}(x) \\
& =u_{C}(x) \wedge \bigvee_{y \otimes z \leq x}\left\{u_{A}^{u_{B}}(y) \wedge u_{B}^{u_{A}}(z)\right\} \\
& =\bigvee_{y \otimes z \leq x}\left\{u_{C}(x) \wedge u_{A}^{u_{B}}(y) \wedge u_{B}^{u_{A}}(z)\right\} \\
& =\bigvee_{\substack{y \otimes z \leq x \\
y \neq 1, z \neq 1}}\left\{u_{C}(x) \wedge u_{A}(y) \wedge u_{C}(x) \wedge u_{B}(z)\right\} \\
& \vee\left\{u_{C}(x) \wedge u_{A}^{u_{B}}(1) \wedge u_{B}(x)\right\} \\
& \vee\left\{u_{C}(x) \wedge u_{A}(x) \wedge u_{B}^{u_{A}}(1)\right\} \\
& =\bigvee_{\substack{y \otimes z \leq x \\
y \neq 1, z \neq 1}}\left\{u_{C}(x) \wedge u_{A}(y) \wedge u_{C}(x) \wedge u_{B}(z)\right\} \\
& \vee\left\{u_{C}(x) \wedge u_{C}(1) \wedge u_{A}^{u_{B}}(1) \wedge u_{B}(x)\right\} \\
& \vee\left\{u_{C}(x) \wedge u_{C}(1) \wedge u_{A}(x) \wedge u_{B}^{u_{A}}(1)\right\} \\
& =\bigvee_{\substack{y \otimes z \leq x \\
y \neq 1, z \neq 1}}\left\{u_{C}(x) \wedge u_{A}(y) \wedge u_{C}(x) \wedge u_{B}(z)\right\} \\
& \vee\left\{u_{C}(1) \wedge\left(u_{A}(1) \vee u_{B}(1)\right)\right. \\
& \left.\wedge\left[\left(u_{C}(x) \wedge u_{B}(x)\right) \vee\left(u_{C}(x) \wedge u_{A}(x)\right)\right]\right\} \\
& =\bigvee_{\substack{y \otimes z \leq x \\
y \neq 1, z \neq 1}}\left\{u_{C}(x) \wedge u_{A}(y) \wedge u_{C}(x) \wedge u_{B}(z)\right\} \\
& \vee\left\{\left[\left(u_{C}(1) \wedge u_{A}(1)\right) \vee\left(u_{C}(1) \wedge u_{B}(1)\right)\right]\right.
\end{aligned}
$$

$$
\begin{aligned}
&\left.\wedge\left[\left(u_{C}(x) \wedge u_{B}(x)\right) \vee\left(u_{C}(x) \wedge u_{A}(x)\right)\right]\right\} \\
& \leq \bigvee_{\substack{y \otimes z \leq x \\
y \neq 1, z \neq 1}}\left\{\left(u_{C} \wedge u_{A}\right)^{u_{C} \wedge u_{B}}(y \vee x)\right. \\
&\left.\wedge\left(u_{C} \wedge u_{B}\right)^{u_{C} \wedge u_{A}}(z \vee x)\right\} \\
& \vee\left[\left(u_{C} \wedge u_{A}\right)^{u_{C} \wedge u_{B}}(1) \wedge\left(u_{C} \wedge u_{A}\right)(y \vee x)\right] \\
& \vee\left[\left(u_{C} \wedge u_{B}\right)^{u_{C} \wedge u_{A}}(1) \wedge\left(u_{C} \wedge u_{B}\right)(z \vee x)\right] \\
&=\bigvee_{y \otimes z \leq x}\left\{\left(u_{C} \wedge u_{A}\right)^{u_{C} \wedge u_{B}}(y \vee x)\right. \\
&\left.\wedge\left(u_{C} \wedge u_{B}\right)^{u_{C} \wedge u_{A}}(z \vee x)\right\} .
\end{aligned}
$$

Let $y \vee x=y^{\prime}$ and $z \vee x=z^{\prime}$; it is easy to verify that $y^{\prime} \otimes z^{\prime} \leq x$, and then the above can be written as

$$
\begin{aligned}
& =\bigvee_{y^{\prime} \otimes z^{\prime} \leq x}\left\{\left(u_{C} \wedge u_{A}\right)^{u_{C} \wedge u_{B}}\left(y^{\prime}\right) \wedge\left(u_{C} \wedge u_{B}\right)^{u_{C} \wedge u_{A}}\left(z^{\prime}\right)\right\} \\
& =\left(u_{C} \wedge u_{A}\right)^{u_{C} \wedge u_{B}} \widetilde{\otimes}\left(u_{C} \wedge u_{B}\right)^{u_{C} \wedge u_{A}}(x), \\
& v_{C}(x) \vee v_{A}^{v_{B}} \widetilde{\odot} v_{B}^{v_{A}}(x) \\
& =v_{C}(x) \vee \bigwedge_{y \otimes z \leq x}\left\{v_{A}^{v_{B}}(y) \vee v_{B}^{v_{A}}(z)\right\} \\
& =\bigwedge_{y \otimes z \leq x}\left\{v_{C}(x) \vee v_{A}^{v_{B}}(y) \vee v_{B}^{v_{A}}(z)\right\} \\
& =\bigwedge_{\substack{y \otimes z \leq x \\
y \neq 1, z \neq 1}}\left\{v_{C}(x) \vee v_{A}(y) \vee v_{C}(x) \vee v_{B}(z)\right\} \\
& \wedge\left\{v_{C}(x) \vee v_{A}^{v_{B}}(1) \vee v_{B}(x)\right\} \\
& \wedge\left\{v_{C}(x) \wedge v_{A}(x) \wedge v_{B}^{v_{A}}(1)\right\} \\
& =\bigwedge_{\substack{y \otimes z \leq x \\
y \neq 1, z \neq 1}}\left\{v_{C}(x) \vee v_{A}(y) \vee v_{C}(x) \vee v_{B}(z)\right\} \\
& \wedge\left\{v_{C}(x) \vee v_{C}(1) \vee v_{A}^{v_{B}}(1) \vee v_{B}(x)\right\} \\
& \wedge\left\{v_{C}(x) \vee v_{C}(1) \vee v_{A}(x) \vee v_{B}^{v_{A}}(1)\right\} \\
& =\bigwedge_{\substack{y \otimes z \leq x \\
y \neq 1, z \neq 1}}\left\{v_{C}(x) \vee v_{A}(y) \vee v_{C}(x) \vee v_{B}(z)\right\} \\
& \wedge\left\{v_{C}(1) \vee\left(v_{A}(1) \wedge v_{B}(1)\right)\right. \\
& \left.\vee\left[\left(v_{C}(x) \vee v_{B}(x)\right) \wedge\left(v_{C}(x) \vee v_{A}(x)\right)\right]\right\} \\
& =\bigwedge_{\substack{y \otimes z \leq x \\
y \neq 1, z \neq 1}}\left\{v_{C}(x) \vee v_{A}(y) \vee v_{C}(x) \vee v_{B}(z)\right\} \\
& \wedge\left\{\left[\left(v_{C}(1) \vee v_{A}(1)\right) \wedge\left(v_{C}(1) \vee v_{B}(1)\right)\right]\right. \\
& \left.\vee\left[\left(v_{C}(x) \vee v_{B}(x)\right) \wedge\left(v_{C}(x) \vee v_{A}(x)\right)\right]\right\}
\end{aligned}
$$




$$
\begin{gathered}
\geq \bigwedge_{\substack{y \otimes z \leq x \\
y \neq 1, z \neq 1}}\left\{\left(v_{C} \vee v_{A}\right)^{v_{C} \vee v_{B}}(y \vee x)\right. \\
\left.\vee\left(v_{C} \vee v_{B}\right)^{v_{C} \vee v_{A}}(z \vee x)\right\} \\
\wedge\left[\left(v_{C} \vee v_{A}\right)^{v_{C} \vee v_{B}}(1) \vee\left(v_{C} \vee v_{A}\right)(y \vee x)\right] \\
\wedge\left[\left(v_{C} \vee v_{B}\right)^{v_{C} \vee v_{A}}(1) \wedge\left(v_{C} \vee v_{B}\right)(z \vee x)\right] \\
=\bigwedge_{y \otimes z \leq x}\left\{\left(v_{C} \vee v_{A}\right)^{v_{C} \vee v_{B}}(y \vee x)\right. \\
\left.\vee\left(v_{C} \vee v_{B}\right)^{v_{C} \vee v_{A}}(z \vee x)\right\} .
\end{gathered}
$$

Let $y \vee x=y^{\prime}$ and $z \vee x=z^{\prime}$; it is easy to verify that $y^{\prime} \otimes z^{\prime} \leq x$, and then the above can be written as

$$
\begin{aligned}
& =\bigwedge_{y^{\prime} \otimes z^{\prime} \leq x}\left\{\left(v_{C} \vee v_{A}\right)^{v_{C} \vee v_{B}}\left(y^{\prime}\right) \vee\left(v_{C} \vee v_{B}\right)^{v_{C} \vee v_{A}}\left(z^{\prime}\right)\right\} \\
& =\left(v_{C} \vee v_{A}\right)^{v_{C} \vee v_{B}} \widetilde{\odot}\left(v_{C} \vee v_{B}\right)^{v_{C} \vee v_{A}}(x) .
\end{aligned}
$$

Thus $u_{C} \wedge u_{A}^{u_{B}} \widetilde{\otimes} u_{B}^{u_{A}} \leq\left(u_{C} \wedge u_{A}\right)^{u_{C} \wedge u_{B}} \widetilde{\otimes}\left(u_{C} \wedge u_{B}\right)^{u_{C} \wedge u_{A}}$ and $v_{C}(x) \vee v_{A}^{v_{B}} \widetilde{\odot} v_{B}^{v_{A}}(x) \geq\left(v_{C} \vee v_{A}\right)^{v_{C} \vee v_{B}} \widetilde{\odot}\left(v_{C} \vee v_{B}\right)^{v_{C} \vee v_{A}}$; that is, the distributivity holds.

\section{Conclusions}

In this paper, by the notion of tip-extended pair of intuitionistic fuzzy sets, it is verified that the set of all intuitionistic fuzzy filters forms a bounded distributive lattice.

Future research will focus on the lattice of fuzzy notions in other algebras by tip-extended pair.

\section{Conflict of Interests}

The author declares that there is no conflict of interests regarding the publication of this paper.

\section{Acknowledgments}

This research was supported by AMEP of Linyi University and the Natural Science Foundation of Shandong Province (Grant no. ZR2013FL006).

\section{References}

[1] M. Ward and R. P. Dilworth, "Residuated lattices," Transactions of the American Mathematical Society, vol. 45, no. 3, pp. 335-354, 1939.

[2] F. Esteva and L. Godo, "Monoidal $t$-norm based logic: towards a logic for left-continuous t-norms," Fuzzy Sets and Systems, vol. 124, no. 3, pp. 271-288, 2001.

[3] P. Hájek, Metamathematics of Fuzzy Logic, Kluwer Academic, Dordrecht, The Netherlands, 1998.

[4] C. C. Chang, "Algebraic analysis of many valued logics," Transactions of the American Mathematical Society, vol. 88, pp. 467490, 1958 .
[5] G. J. Wang, An Introduction to Mathematical Logic and Resolution Principle, Science in China Press, Beijing, China, 2003.

[6] E. Turunen, Mathematics Behind Fuzzy Logic, Physica, Heidelberg, Germany, 1999.

[7] B. van Gasse, G. Deschrijver, C. Cornelis, and E. E. Kerre, "Filters of residuated lattices and triangle algebras," Information Sciences, vol. 180, no. 16, pp. 3006-3020, 2010.

[8] E. Turunen, "Boolean deductive systems of BL-algebras," Archive for Mathematical Logic, vol. 40, no. 6, pp. 467-473, 2001.

[9] M. Kondo, "Filters on commutative residuated lattices," in Integrated Uncertainty Management and Applications, vol. 68, pp. 343-347, Springer, Berlin, Germany, 2010.

[10] L. Liu and K. Li, "Boolean filters and positive implicative filters of residuated lattices," Information Sciences, vol. 177, no. 24, pp. 5725-5738, 2007.

[11] Y. Zhu and Y. Xu, "On filter theory of residuated lattices," Information Sciences, vol. 180, no. 19, pp. 3614-3632, 2010.

[12] M. Haveshki, A. B. Saeid, and E. Eslami, "Some types of filters in BL algebras," Soft Computing, vol. 10, no. 8, pp. 657-664, 2006.

[13] M. Kondo and W. A. Dudek, "Filter theory of BL algebras," Soft Computing, vol. 12, no. 5, pp. 419-423, 2008.

[14] A. B. Saeid and S. Motamed, "Normal filters in BL-algebras," World Applied Sciences Journal, vol. 7, pp. 70-76, 2009.

[15] E. Turunen, "BL-algebras of basic fuzzy logic," Mathware \& Soft Computing, vol. 6, no. 1, pp. 49-61, 1999.

[16] A. Rosenfeld, “Fuzzy groups," Journal of Mathematical Analysis and Applications, vol. 35, no. 3, pp. 512-517, 1971.

[17] L. A. Zadeh, "Fuzzy sets," Information and Control, vol. 8, no. 3, pp. 338-353, 1965.

[18] J. N. Mordeson and D. S. Malik, Fuzzy Commutative Algebra, World Scientific, London, UK, 1998.

[19] T. Head, "A metatheorem for deriving fuzzy theorems from crisp versions," Fuzzy Sets and Systems, vol. 73, no. 3, pp. 349358,1995

[20] T. Head, "Erratum to "A metatheorem for deriving fuzzy theorems from crisp versions"' Fuzzy Sets and Systems, vol. 79, no. 2, pp. 277-278, 1996.

[21] I. Jahan, "The lattice of $L$-ideals of a ring is modular," Fuzzy Sets and Systems, vol. 199, pp. 121-129, 2012.

[22] Y. B. Jun, Y. Xu, and X. H. Zhang, "Fuzzy filters of MTLalgebras," Information Sciences, vol. 175, no. 1-2, pp. 120-138, 2005.

[23] L. Liu and K. Li, "Fuzzy filters of BL-algebras," Information Sciences, vol. 173, no. 1-3, pp. 141-154, 2005.

[24] J. L. Zhang and H. J. Zhou, "Fuzzy filters on the residuated lattices," New Mathematics and Natural Compution, vol. 2, no. 1, pp. 11-28, 2006.

[25] K. T. Atanassov, "Intuitionistic fuzzy sets," Fuzzy Sets and Systems, vol. 20, no. 1, pp. 87-96, 1986.

[26] Z. Xue, Y. Xiao, W. Liu, H. Cheng, and Y. Li, "Intuitionistic fuzzy filter theory of BL-algebras," International Journal of Machine Learning and Cybernetics, vol. 4, no. 6, pp. 659-669, 2013. 


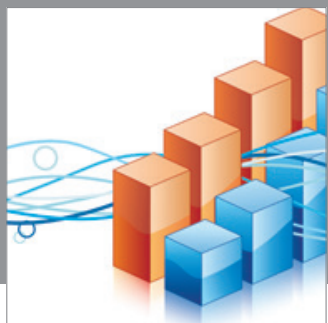

Advances in

Operations Research

mansans

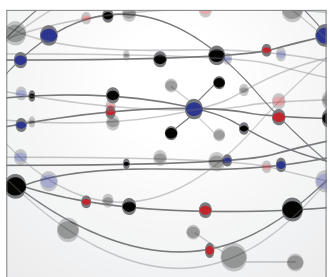

The Scientific World Journal
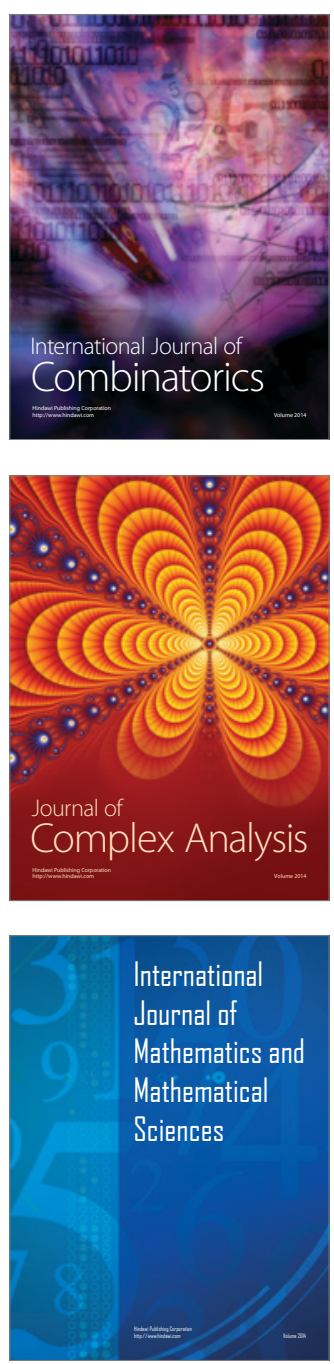
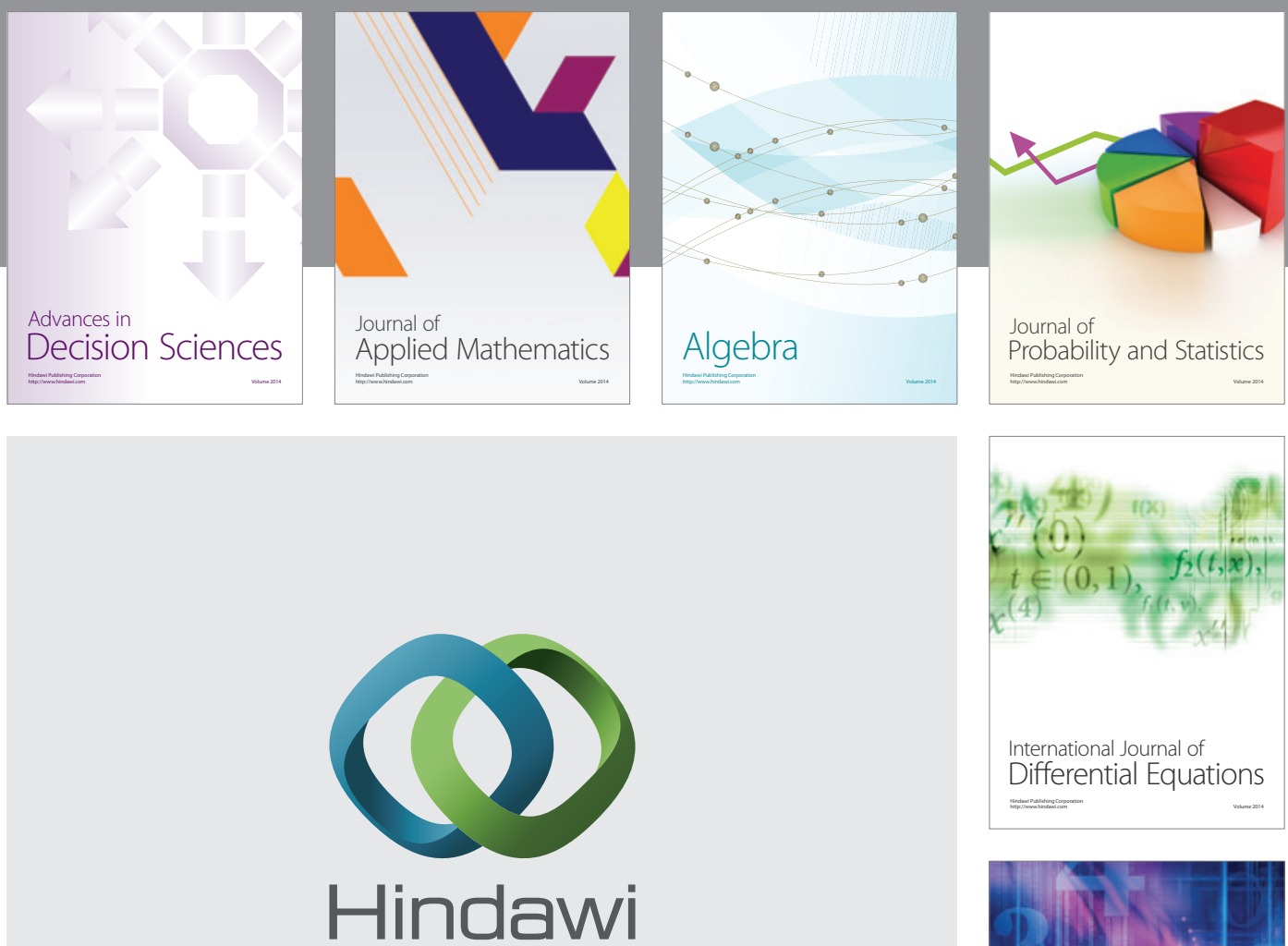

Submit your manuscripts at http://www.hindawi.com
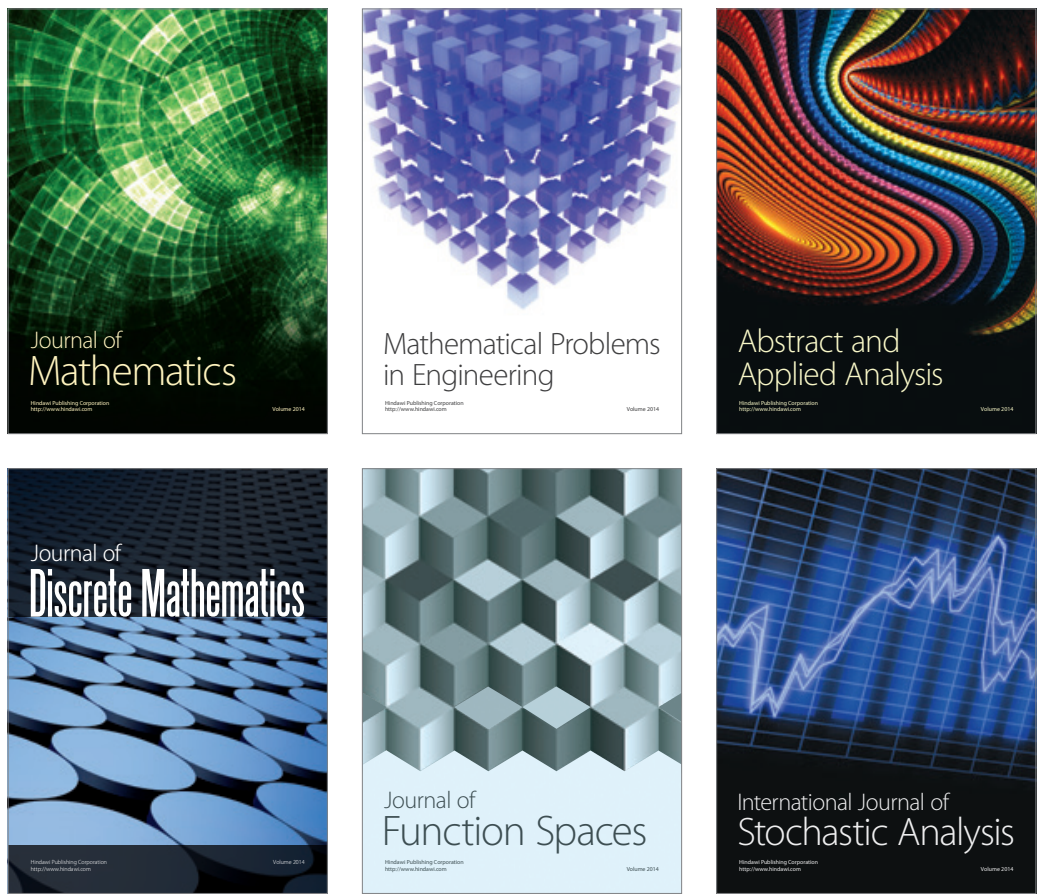

Journal of

Function Spaces

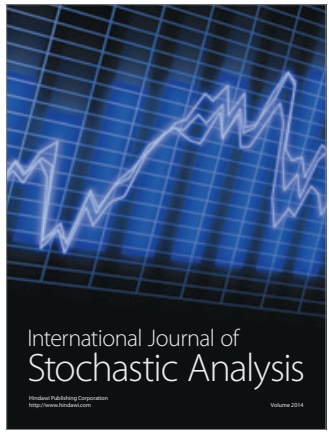

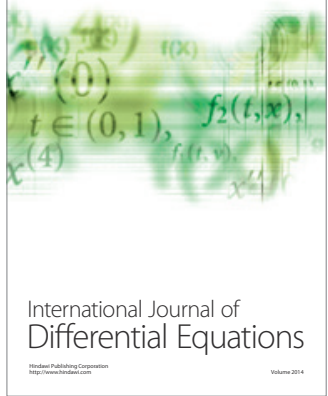
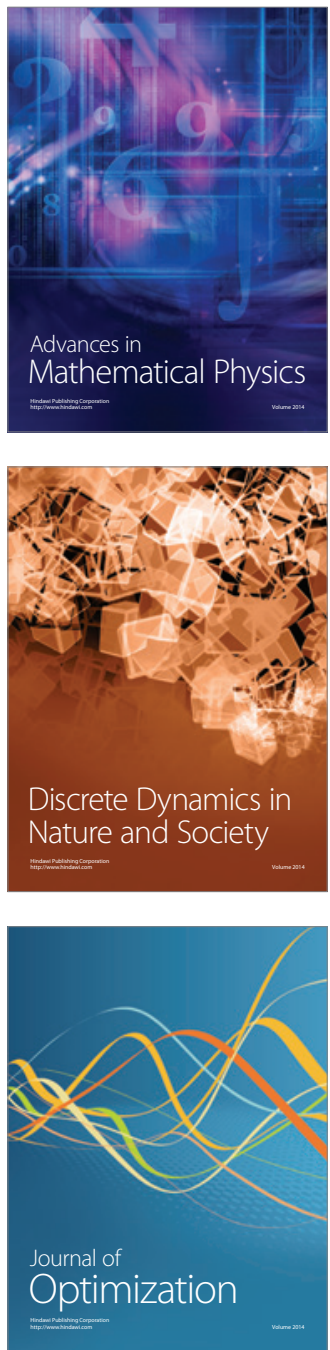